\title{
Antihypertensive treatment in critical limb ischaemia
}

\author{
Anders Gottsäter, Peter M. Nilsson \\ University of Lund, Department of Vascular Diseases, Skåne University Hospital
}

\begin{abstract}
Peripheral artery disease (PAD) is defined as atherosclerotic arterial occlusive disease of the lower extremities, manifesting as intermittent claudication (IC, pain induced by walking) or critical limb ischaemia (CLI, rest pain or ulcerations). PAD guidelines recommend strict control of cardiovascular risk factors, and European guidelines on hypertension recommend a blood pressure (BP) target $<140 / 90 \mathrm{~mm} \mathrm{Hg}$ also in PAD patients. As the pressure in the affected extremity might be of relevance for the prognosis concerning limb salvage in CLI, the traditional approach was to avoid beta-blockers and allow a slightly higher BP in CLI. Both theoretical considerations and observational data support aggressive BP lowering also in CLI; however, in the absence of randomized studies on BP lowering in this setting it cannot be definitely established that current recommendations on BP lowering apply also in CLI. key words: peripheral artery disease, critical limb ischaemia, blood pressure
\end{abstract}

\section{Hypertension in peripheral arterial disease}

Peripheral artery disease (PAD) is defined as atherosclerotic arterial occlusive disease of the lower extremities. PAD can manifest as intermittent claudication (IC, pain induced by walking) or critical limb ischaemia (CLI, rest pain or ulceration for more than 14 days) [1]. PAD affected 202 million people worldwide in 2010 [2,3], and in a database of over 9 million patients, PAD prevalence was $10.7 \%$ [4]. Furthermore, PAD patients have a high frequency of concomitant atherosclerotic disease, and risk factors for atherosclerosis such as arterial hypertension are common in this population [5, 6]. Major PAD guidelines issued by ACC/AHA [7] and TASC [1], recommend strict control of cardiovascular risk factors in this group of patients, and the current European guidelines on treatment of hypertension recommend a blood pressure (BP) target of less than 140/90 $\mathrm{mm} \mathrm{Hg}$ also in PAD patients [8].

Prior concerns on blood pressure treatment and beta-blocker use in IC patients have been offset by meta-analyses from the Cochrane Collaboration [9-11]. This work has confirmed that the benefits of treating hypertension in PAD patients are overwhelming, whereas there is no clear evidence that any antihypertensive drug class confers specific benefits in relation to another [9-11]. The vast majority of patients in the studies in the above meta-analyses have, however, been included in studies with a diagnosis of IC. Patients with CLI represent about $1 \%$ of the total number of PAD patients [1], and as this condition is scientifically defined as chronic ischaemic rest pain, ulcers, or gangrene attributable to objectively proven arterial occlusive disease (ankle pressure of $<50-70 \mathrm{~mm} \mathrm{Hg}$, or toe pressure of $<30-50 \mathrm{~mm} \mathrm{Hg}$, or $\mathrm{TCPO}_{2}<30-50 \mathrm{~mm} \mathrm{Hg}$ ) [1],

Address for correspondence: Prof. Anders Gottsäter

University of Lund, Department of Vascular Diseases, Skåne University Hospital

S-205 02 Malmö, Sweden

tel. +46 40331000 , fax +46 40338097

e-mail: anders.gottsater@med.lu.se 
inclusion and follow-up of these patients in scientific studies of blood pressure lowering is complicated. Therefore, most review authors do not address this group specifically.

\section{Antihypertensive treatment in critical limb ischaemia}

Furthermore, blood pressure might have other relevance in CLI than as a risk factor for cardiovascular disease. As the pressure in the affected extremity might be of relevance for the prognosis concerning limb salvage, the traditional approach was to avoid beta-blockers and allow a slightly higher blood pressure in this setting [12]. Additionally, the concept of increasing blood pressure and thereby increasing collateral circulation in an ischaemic extremity seems intuitionally appealing. Regional hyperperfusion via extracorporeal limb perfusion has been evaluated in CLI with promising results [13], and benefits of sequential pneumatic compression have been suggested in prevention of minor amputation, prolonging amputation-free survival, and improving rest pain in patients with non-reconstructable CLI [14].

Many factors would theoretically support a benefit of aggressive blood pressure lowering also in CLI, however. High frequencies of concomitant coronary heart disease $[15,16]$ congestive heart failure [16-18], and coronary valve disease [19] have been demonstrated in this group. In a necropsy study [16], $92 \%$ of patients amputated because of CLI showed advanced coronary atherosclerosis. Five-year cardiovascular mortality in symptomatic PAD in different studies was recently estimated to $13 \%$ (CI 9-17\%) by Sigvant et al. [20]. In CLI, this figure is higher — around 20\% already after one year [21-23] and mainly caused by cardiac disease [21-23].

Blood pressure seems to be of relevance for this outcome. Increased preoperative pulse pressure [24] and uncontrolled hypertension [25] are associated with procedural complications and increased mortality in patients after distal intervention for CLI. In other studies of primary revascularization in CLI patients, lack of beta-blocking therapy has been associated with increased risk of heart related adverse events [26, 27].

\section{Conclusion}

Although the above observational data from CLI patients supports recommendations on antihypertensive treatment in CLI, it cannot be definitely established that current recommendations on antihypertensive treatment apply also in this group in the absence of randomized studies on blood pressure lowering. Patients with CLI therefore need to be further studied by physicians with knowledge of and interest for treatment of arterial hypertension. As different relationships between blood pressure levels and tissue perfusion might apply in diabetic and non-diabetic patients with limb ischaemia [28, 29], these two groups should preferably be studied separately.

\section{References}

1. Norgren L., Hiatt W., Dormandy J., Nehler M., Harris K., Fowkes F. Inter-Society Consensus for the Management of Peripheral Arterial Disease (TASC II). J. Vasc. Surg. 2007; 45 (Suppl. 1): S5-S67.

2. Fowkes F.G., Rudan D., Rudan I. et al. Comparison of global estimates of prevalence and risk factors for peripheral artery disease in 2000 and 2010: a systematic review and analysis. Lancet 2013; 382: $1329-1340$.

3. Criqui M.H., Aboyans V. Epidemiology of peripheral artery disease. Circ. Res. 2015; 116: 1509-1526.

4. Nehler M.R., Duval S., Diao L. et al. Epidemiology of peripheral arterial disease and critical limb ischemia in an insured national population. J. Vasc. Surg. 2014; 60: 686-695.

5. Aronow W.S., Ahn C. Prevalence of coexistence of coronary artery disease, peripheral arterial disease, and atherothrombotic brain infarction in men and women $>$ or $=62$ years of age. Am. J. Cardiol. 1994; 74: 64-65.

6. Ness J., Aronow W.S. Prevalence of coexistence of coronary artery disease, ischemic stroke, and peripheral arterial disease in older persons, mean age 80 years, in an academic hospital-based geriatrics practice. J. Am. Geriatr. Soc. 1999; 47: 1255-1256.

7. Hirsch A.T., Haskal Z.J., Hertzer N.R. et al. ACC/AHA 2005 Practice Guidelines for the management of patients with peripheral arterial disease (lower extremity, renal, mesenteric, and abdominal aortic): a collaborative report from the American Association for Vascular Surgery/Society for Vascular Surgery, Society for Cardiovascular Angiography and Interventions, Society for Vascular Medicine and Biology, Society of Interventional Radiology, and the ACC/AHA Task Force on Practice Guidelines (Writing Committee to Develop Guidelines for the Management of Patients With Peripheral Arterial Disease): endorsed by the American Association of Cardiovascular and Pulmonary Rehabilitation; National Heart, Lung, and Blood Institute; Society for Vascular Nursing; TransAtlantic Inter-Society Consensus. Circulation 2006; 113: e463-654.

8. Mancia G., Fagard R., Narkiewicz K. et al. 2013 ESH/ESC guidelines for the management of arterial hypertension: the task force for the management of arterial hypertension of the European Society of Hypertension (ESH) and of the European Society of Cardiology (ESC). Eur. Heart J. 2013; 34: 2159-2219.

9. Lane D.A., Lip G.Y. Treatment of hypertension in peripheral arterial disease. Cochrane Database Syst. Rev. 2013 Dec 4; (12): CD003075. doi: 10.1002/14651858.CD003075.pub3.

10. Paravastu S.C., Mendonca D.A., Da Silva A. Beta blockers for peripheral arterial disease. Cochrane Database Syst. Rev. 2009 Oct 7; (4): CD003075. doi: 10.1002/14651858.CD003075.pub2.

11. Lane D.A., Lip G.Y. Treatment of hypertension in peripheral arterial disease. Cochrane Database Syst. Rev. 2013 Dec 4; (12): CD003075. doi: 10.1002/14651858.CD003075.pub3.

12. Diehm C. Antihypertensive therapy in arterial occlusive disease. Vasa Suppl. 1991; 33: 71-74.

13. Khin N.Y., Dijkstra M.L., Huckson M. et al. Hypertensive extracorporeal limb perfusion for critical limb ischemia. J. Vasc. Surg. 2013; 58: 1244-1253. 
14. Zaki M., Elsherif M., Tawfick W., El Sharkawy M., Hynes N., Sultan $S$. The role of sequential pneumatic compression in limb salvage in non-reconstructable critical limb ischemia. Eur. J. Vasc. Endovasc. Surg. 2016; 51: 565-571.

15. Fowkes F.G. Epidemiology of atherosclerotic arterial disease in the lower limbs. Eur. J. Vasc. Surg. 1988; 2: 283-291.

16. Mautner G.C., Mautner S.L., Roberts W.C. Amounts of coronary arterial narrowing by atherosclerotic plaque at necropsy in patients with lower extremity amputation. Am. J. Cardiol. 1992; 70: 1147-1151.

17. Aronow W.S., Ahn C. Prevalence of coexistence of coronary artery disease, peripheral arterial disease, and atherothrombotic brain infarction in men and women $>$ or $=62$ years of age. Am. J. Cardiol. 1994; 74: 64-65.

18. Ness J., Aronow W.S. Prevalence of coexistence of coronary artery disease, ischemic stroke, and peripheral arterial disease in older persons, mean age 80 years, in an academic hospital-based geriatrics practice. J. Am. Geriatr. Soc. 1999; 47: 1255-1256.

19. Aronow W.S., Ahn C., Kronzon I. Association of valvular aortic stenosis with symptomatic peripheral arterial disease in older persons. Am. J. Cardiol. 2001; 88: 1046-1047.

20. Sigvant B., Lundin F., Wahlberg E. The risk of disease progression in peripheral arterial disease is higher than expected: A meta-analysis of mortality and disease progression in peripheral arterial disease. Eur. J. Vasc. Endovasc. Surg. 2016; 51: 395-403.

21. Wolfe J. Defining the outcome of critical ischaemia: a one year prospective study. Br. J. Surg. 1986; 73: 321.

22. Long-term mortality and its predictors in patients with critical leg ischaemia. The I.C.A.I. Group (Gruppo di Studio dell'Ischemia Cronica Critica degli Arti Inferiori). The Study Group of Criticial
Chronic Ischemia of the Lower Exremities. Eur. J. Vasc. Endovasc. Surg. 1997; 14: 91-95.

23. Barani J., Nilsson J.A., Mattiasson I., Lindblad B., Gottsäter A. Inflammatory Mediators are associated with 1-year mortality in critical limb ischemia. J. Vasc. Surg. 2005; 42: 75-80.

24. Zettervall S.L., Buck D.B., Darling J.D., Lee V., Schermerhorn M.L., Guzman R.J. Increased preoperative pulse pressure predicts procedural complications and mortality in patients who undergo tibial interventions for critical limb ischemia. J. Vasc. Surg. 2016; 63: 673-677.

25. Im S.I., Rha S.W., Choi B.G. et al. Impact of uncontrolled hypertension on 12-month clinical outcomes following below-the-knee arteries (BTK) interventions in patients with critical limb ischemia. Clin. Hypertens. 2016; 29; 22-29.

26. Conte M.S., Bandyk D.F., Clowes A.W., Moneta G.L., Namini H., Seely L. Risk factors, medical therapies and perioperative events in limb salvage surgery: observations from the PREVENT III multicenter trial. J. Vasc. Surg. 2005; 42: 456-464.

27. Flu H.C., Lardenoye J.H., Veen E.J., Aquarius A.E., Van Berge Henegouwen D.P., Hamming J.F. Morbidity and mortality caused by cardiac adverse events after revascularization for critical limb ischemia. Ann. Vasc. Surg. 2009; 23: 583-597.

28. Wahlberg E., Jörneskog G. Patients with diabetes and critical limb ischemia have a high peripheral vascular resistance. Ann. Vasc. Surg. 1997; 11: 224-229.

29. Jörneskog G. Why critical limb ischemia criteria are not applicable to diabetic foot and what the consequences are. Scand. J. Surg. 2012; 101: 114-118. 